\title{
STIMULATED SCATTERING OF MICROCAVITY POLARITONS
}

\author{
J. Bloch, P. Senellart, V. Thierry-Mieg and J.Y. Marzin \\ Laboratoire de Microstructures et de Microélectronique, 196 av. Henri Ravéra \\ 92225 Bagneux Cedex, France
}

\begin{abstract}
We report on cw optical experiments performed in a semiconductor microcavity containing a single quantum well in the strong coupling regime. Angularly resolved photoluminescence measurements under non-resonant excitation show the collapse of a relaxation bottleneck as the excitation power is increased. As a result, the emission close to $k_{\|}=0$ presents a non-linear behavior. In a two-beam experiment we resonantly inject polaritons at $k_{\|}=0$ and show that relaxation from states with large in-plane wave vector toward $k_{\|}=0$ is stimulated by the polariton final state population.
\end{abstract}

PACS numbers: $71.36 .+\mathrm{c}, 71.35 . \mathrm{Lk}, 78.30 . \mathrm{Fs}, 42.55 . \mathrm{Sa}$

\section{Introduction}

In a semiconductor microcavity containing quantum wells, both excitons and photons are two-dimensional (confined along the growth direction and free of motion in the perpendicular plane). As a result, the dipolar interaction responsible for the radiative coupling between excitons and photons couples each exciton with a given in-plane wave vector $k_{\|}$with a single photon mode of the same $k_{\|}$. This one-to-one state coupling gives rise to the formation of mixed exciton-photon eigenstates, also named cavity polaritons in analogy to bulk material polaritons. This strong coupling regime in a semiconductor microcavity has been first evidenced in 1992 by Weisbuch et al. [1] and has been the subject of intense studies these last years [2].

Because of the formation of this mixed exciton-photon eigenstates, the light emission inside the cavity is not governed by the Fermi golden rule and the front emission detected from outside the sample is due to leakage of the polariton photon part through the top mirror. Therefore in the strong coupling regime, photon stimulated emission can no longer occur. However another non-linear mechanism can be considered. One of the unique features of photon-like cavity polaritons is their extremely small in-plane effective mass (typically $10^{-5} m_{0}$ ). Because of this reduced density of states, occupation factors close to one may be reached before bleaching of the exciton. It has actually been predicted that relaxation processes such as phonon scattering [3] or exciton-exciton collisions [4] could be stimulated 
by the polariton final state population. This would give rise to a non-linear increase in the emission induced by a mechanism of fundamentally different nature than the lasing emission of a standard system in the weak coupling regime. This non-linear behavior would be the signature of the quasi-bosonic nature of cavity polaritons and excitons. However high carrier densities are required to trigger scattering stimulation but need to be small enough for phase space filling and screening to be negligible and exciton Bose commutator to be close to 1 [5]. The bleaching of the strong coupling regime is indicated by a broadening and a spectral shift of the polariton modes towards the uncoupled modes $[6,7]$.

Non-linear emission under non-resonant excitation has been first reported in II-VI microcavities $[8,9]$ where the exciton oscillator strength is larger than in III-V compounds so that the exciton bleaching occurs for larger carrier densities $\left(\approx 10^{13} \mathrm{~cm}^{-2}\right)$. Lately we reported similar observations in a III-V heterostructure [10]. Since these first measurements, many studies have been devoted to trying to elucidate the role of the bosonic nature of polaritons in these observations [11-16]. In particular, pulsed two beam experiments have shown that when pumping the system at a given in-plane wave vector and probing at $k=0$, it is possible to stimulate the polariton-polariton scattering: gain can be seen on the reflected probe beam [12], or an increase in the upper branch emission is observed [13]. These experiments were mostly done under pulsed and resonant excitation.

In this paper we give a closer insight into the non-linearity under non-resonant excitation we previously reported. We first report on angle resolved photoluminescence (PL) experiments under $\mathrm{cw}$ non-resonant excitation evidencing the collapse of the relaxation bottleneck when increasing excitation power. We then report on recent two-beam experiments: an additional $\mathrm{cw}$ probe beam resonantly injects polaritons into states close to $k=0$. We observe gain on the probe beam in reflectivity measurements simultaneously to a decrease in larger $k$ state emission. This is the unambiguous signature of the stimulation of scattering by the final state population injected by the probe beam.

\section{Sample structure and experimental setup}

The sample has been grown by molecular beam epitaxy. It consists of a $\lambda$ GaAs cavity containing a single InAs quantum well at the center, and surrounded by two Bragg mirrors. The top (bottom) mirror contains 20 (24) $\mathrm{Ga}_{0.9} \mathrm{Al}_{0.1} \mathrm{As} / \mathrm{AlAs}$ $\lambda / 4$ layers. Because of the spatial variation of the layer thickness along the wafer, different detunings $\delta=E_{\mathrm{c}}-E_{\mathrm{x}}$ between the cavity mode of energy $E_{\mathrm{c}}$ and the exciton of energy $E_{\mathrm{x}}$ can be probed by changing the spot on the sample.

Reflectivity performed at $1.4 \mathrm{~K}$ show the anticrossing between the exciton and the cavity mode, with a Rabi splitting of $3.5 \mathrm{meV}$. At resonance, the upper (lower) polariton line present a width of $0.51 \mathrm{meV}(0.23 \mathrm{meV})$, highlighting the high optical quality of the sample.

Photoluminescence experiments are performed at $1.4 \mathrm{~K}$ with either a single or two excitation beams. Two cw Ti:sapphire laser beam are coupled to optical fibers of $200 \mu \mathrm{m}$ core and imaged on the sample into the same $100 \mu \mathrm{m}$ diameter spot (see Fig. 1). The emission of a $40 \mu \mathrm{m}$ diameter area within the excited spot is collected, dispersed through a monochromator and detected with either a multichannel Si 


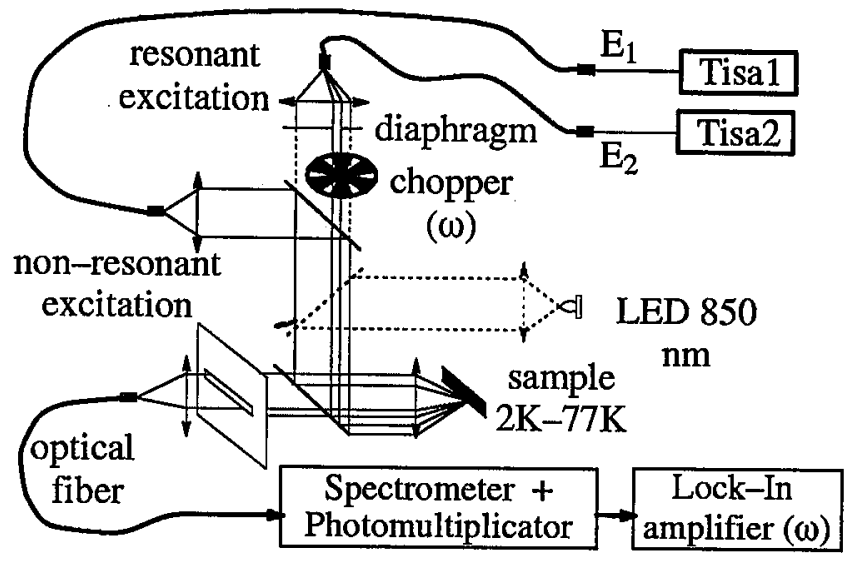

Fig. 1. Schematic of the experimental setup.

detector or a GaAs photomultiplier. For modulated experiments, we use the GaAs photomultiplier and a lock-in amplifier.

\section{Luminescence under non-resonant excitation}

We report first on luminescence measurements performed with a single non-resonant excitation beam tuned to $1.6 \mathrm{eV}$. The sample is tilted so that its normal crosses the very edge of the collecting lens. We first collect the emission through a small aperture scanned from normal incidence to an angle of 25 degrees. Figure 2 shows the PL energy of the two polariton lines as a function of the detection angle for a detuning $\delta=-5 \mathrm{meV}$. As first shown by Houdré et al. [17], polaritons of different in-plane wave vector emit photons with different external angles so that angularly resolved emission measurements give insight into the in-plane

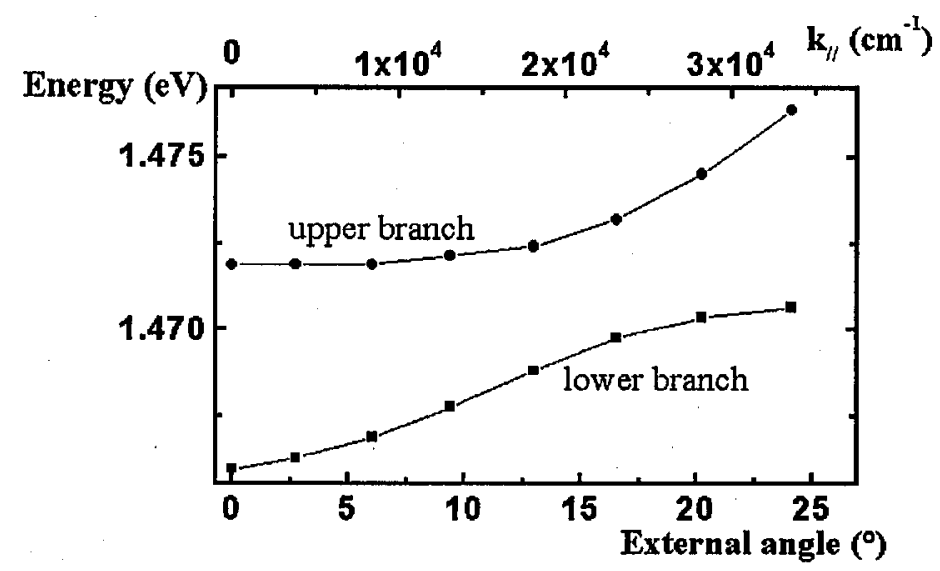

Fig. 2. Energy of the polariton lines measured for a detuning $\delta=-5 \mathrm{meV}$ at $2 \mathrm{~K}$ by photoluminescence as a function of the detected angle. Also indicated the corresponding in-plane wave vector $k_{\|}$. 


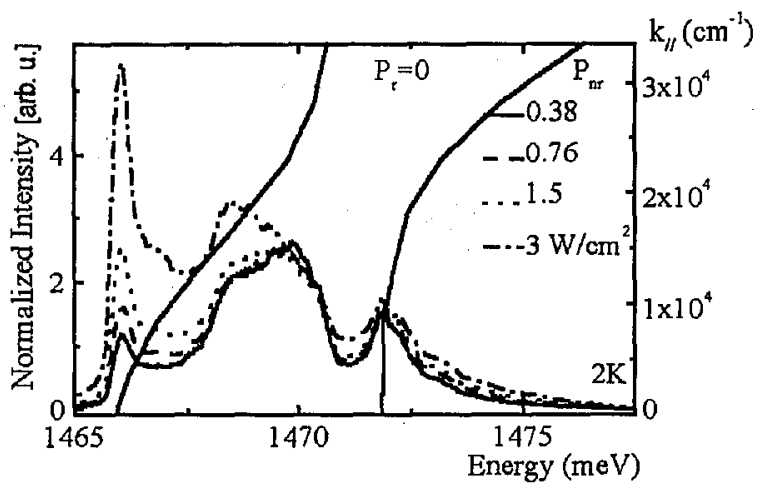

Fig. 3. Photoluminescence spectra normalized to the excitation power measured for a detuning $\delta=-5 \mathrm{meV}$ through the rectangular aperture for different excitation powers.

dispersion of the two polariton lines. We now collect the emission through a rectangular aperture (see Fig. 1), thus simultaneously detecting the emission with angles ranging from $0^{\circ}$ to $22^{\circ}$ with respect to normal incidence. That way we measure the emission of all polaritons with in-plane wave vector ranging from 0 to $3 \times 10^{4} \mathrm{~cm}^{-1}$. Figure 3 shows emission spectra normalized to the excitation power measured through this rectangular aperture for various excitation powers. Emission from polaritons of different wave vectors are discriminated according to their emission energy. For clarity the different in-plane wave vectors deduced from Fig. 1 are shown in the same figure. For small excitation powers, polaritons accumulate close to $k_{\|}=2 \times 10^{4} \mathrm{~cm}^{-1}$ and the signal coming from polaritons of $k=0$ is smaller. Because of the strong in-plane dispersion and of the photon character of $k_{\|}=0$ polaritons, the polariton interaction with acoustic phonons is inhibited and this results in a relaxation bottleneck, evidenced by these measurements. The system is not in thermal equilibrium. As we increase the excitation power, the signal arising from polaritons close to $k_{\|}=0$, presents a clear non-linear increase. Simultaneously the signal from polaritons of $k_{\|}=2 \times 10^{4} \mathrm{~cm}^{-1}$ or from the upper branch present a linear behavior, except for the highest excitation power. This shows that as we increase the excitation power, relaxation through polariton states of $k_{\|}=0$ is modified and becomes much more efficient. The relaxation bottleneck vanishes with increasing excitation power. Several mechanisms can be responsible for the improvement of the relaxation efficiency. As carrier density increases with the excitation power, exciton-exciton or polariton-polariton interaction become more efficient and accelerate relaxation processes. Actually it has been shown in Ref. [12] that polariton-polariton interaction is the stimulated scattering responsible for the large gain observed. A signature of this two-particle mechanism in this experiment is the very narrow window in $k_{\|}$space where gain is observed, a window corresponding to the conservation of both wave vector and energy during the collision process.

As the excitation power is increased, relaxation toward $k_{\|}=0$ becomes more efficient and the population close to $k_{\|}=0$ increases. If the occupation factor of these states becomes larger than one, relaxation toward these states could be stimulated by the final state population. Recently we have measured the absolute 
intensity of the emission in order to estimate the actual number of photons emitted by the sample and as a result the polariton population as a function of $k_{\|}$[18]. We show that at the onset of the non-linearity, the polariton occupation factor is very small (of the order of $10^{-3}$ ) and the non-linear behavior is the result of more efficient polariton-polariton interactions. For higher excitation power and before the strong coupling regime bleaching begins, occupation factors larger than one (of the order of 5) are reached. At this stage, stimulation of the relaxation processes can occur but can be hardly evidenced in such a simple non-resonant measurement.

\section{Two-beam experiment}

To evidence a stimulated process, we perform a $\mathrm{cw}$ two-beam experiment where the sample is simultaneously excited with a non-resonant beam as in the previous part and resonantly excited with a second beam under normal incidence. In a first experiment, the non-resonant pump is chopped at low frequency $\omega=$ $560 \mathrm{~Hz}$ (see Fig. 4b). We scan the resonant beam across the lower polariton line and measure both the continuous component and the modulated component of the reflected probe beam. We extract the relative change of the reflectivity for various non-resonant excitation powers. For experimental reasons not detailed here, we independently measure the reflectivity of the sample without the pump beam with a light emitting diode emitting around $850 \mathrm{~nm}$ and deduce the reflectivity spectra in the presence of the pump beam.
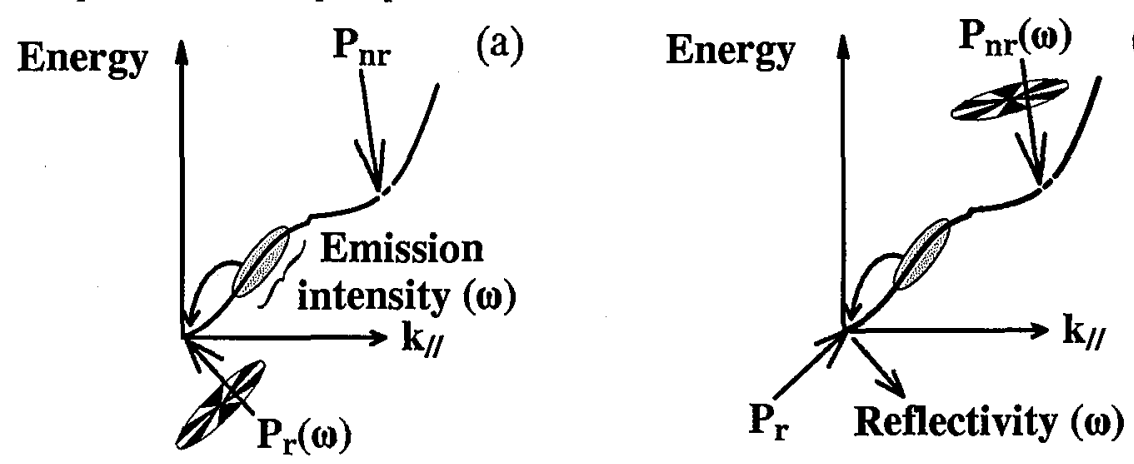

Fig. 4. Principle of the two-beam experiment: (a) we modulate the probe beam and measure the modulated component of the emission of large $k_{\|}$state; (b) we modulate the non-resonant pump beam and monitor the modulated component of the reflected probe beam.

Figure 5 shows the reflectivity spectra of the lower polariton branch measured for different non-resonant excitation powers and for two detunings. For both detunings, the reflectivity dip gets significantly more pronounced as the non-resonant power is increased. Moreover, no significant shift of the line is observed for power densities up to $60 \mathrm{~W} / \mathrm{cm}^{2}$, showing that the system remains in the strong coupling regime.

This behavior is the signature of gain inside the cavity, and can be understood qualitatively as follows: the reflected intensity is the result of destructive 


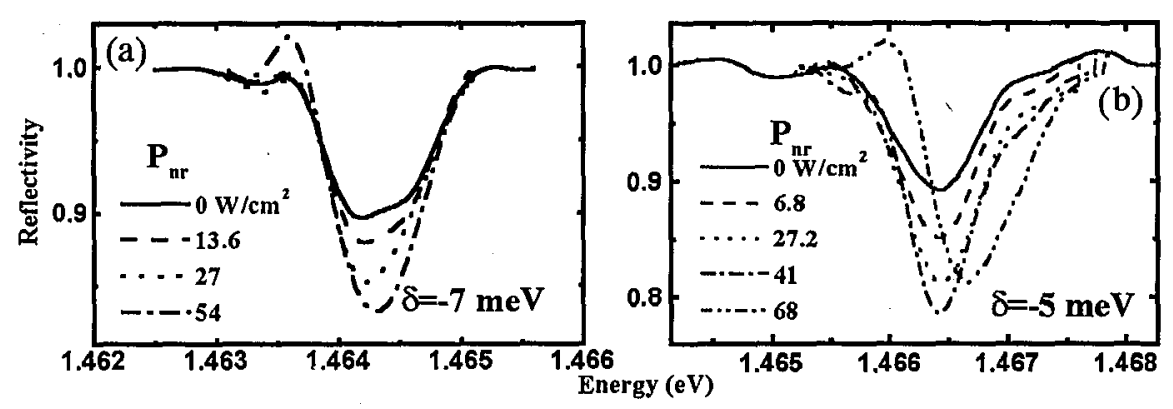

Fig. 5. Reflectivity spectra measured for a detuning (a) $\delta=-7 \mathrm{meV}$ and (b) $\delta=$ $-5 \mathrm{meV}$ and for various non-resonant excitation powers $P_{\mathrm{nr}}$.

interference between light reflected on the surface and light that has propagated inside the cavity. If the amplitude of the field that has propagated inside the cavity, becomes larger, then on one hand the intensity of the reflected beam decreases, a result of more efficient destructive interference, and on the other hand the intensity of the transmitted beam increases.

Actually, in the regime where the reflectivity dip becomes more pronounced, we have checked experimentally that the transmission line becomes more intense by the same amount [18]. Of course, the presence of gain results in a decrease in the reflected intensity only in the limit of small gains. If the gain was much larger, then reflectivity even larger than one should be observed as in Ref. [12]. Such a high gain regime could not be achieved by increasing the non-resonant excitation power. Indeed in our experiment, before achieving a large enough gain, the strong coupling regime bleaching starts. The main difference with Ref. [12, 1.3] (where a large polariton population was directly created at a given in-plane wave vector) is that in our case we use a non-resonant excitation. As a result we create a carrier population over a large range of $k_{\|}$states for which the polariton-polariton scattering toward $k_{\|}=0$ is less efficient than for the specific angle used in Ref. [12].

Let us come back to the physical origin of the gain we measure. We think that it arises from polariton scattering stimulated by the polariton field injected by the resonant beam. If we consider the amplitude of the field of the resonant beam for an excitation power of $0.4 \mathrm{~W} / \mathrm{cm}^{2}$ focused onto a spot size of $100 \mu \mathrm{m}$, it corresponds to a polariton occupation factor of the order of 10. In this experiment, the system is in a interesting regime where small occupation factors of all states from the polariton lower branch are created by the non-resonant beam, and the resonant beam locally creates an occupation factor much larger than one. Thanks to the small non-resonant excitation power, we can expect the polaritons to be close to the bosonic regime.

Figure 6 shows how the reflectivity dip, and as a result the gain, varies with the non-resonant excitation power for the two investigated detunings. The gain increases with the pump power and is larger for a more exciton-like polariton.

To further evidence that the resonant beam stimulates the scattering of polaritons with larger in-plane wave vectors toward $k_{\|}=0$, we now look at the emission of the large $k_{\|}$polariton states in the presence of the probe beam. As 


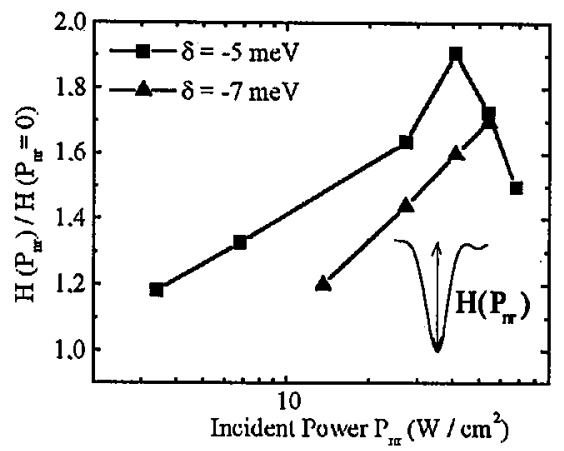

Fig. 6. Normalized height of the reflectivity dip as a function of the non-resonant excitation power for two detunings $\delta$.

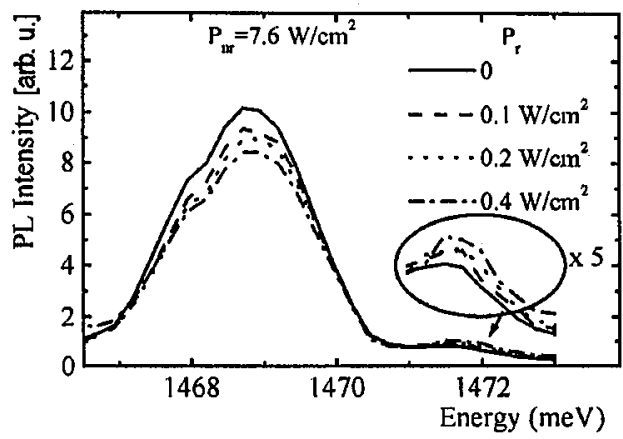

Fig. 7. PL spectra measured for $\delta=-5 \mathrm{meV}$ under a non-resonant excitation power of $7.6 \mathrm{~W} / \mathrm{cm}^{2}$ and various non-resonant powers $P_{\mathrm{r}}$.

in the previous part, we collect the emission through a rectangular aperture and look at emission with an external angle ranging from 0 to $22^{\circ}$. We mask the region close to $k_{\|}=0$ to protect the detector from the reflected resonant beam. The probe beam is now chopped at frequency $\omega$, and we measure the modulated component $\triangle \mathrm{PL}$ of the emission from 8 to $22^{\circ}$ (see Fig. 4a). Figure 7 shows the photoluminescence spectrum in the presence of only the non-resonant beam. The intensity is peaked around $1469 \mathrm{meV}$, corresponding to states with $k_{\|}$of the order of $1.5 \times 10^{4} \mathrm{~cm}^{-1}$, again showing the relaxation bottleneck. Photoluminescence spectra deduced from $\triangle \mathrm{PL}$ measurements in the presence of the resonant beam are also shown for various probe powers. We observe a significant decrease in the luminescence signal around $1469 \mathrm{meV}$ with increasing resonant power. This shows that the resonant beam in $k_{\|}=0$ induces a reduction of the population of polariton states close to $k_{\|}=1.5 \times 10^{4} \mathrm{~cm}^{-1}$. This is the signature of a stimulation of the polariton relaxation by the final state population close to $k_{\|}=0$. Simultaneously to the decrease in the lower branch luminescence, we observe an increase in the upper branch emission. This indicates that one possible stimulated mechanism is a polariton-polariton interaction, one polariton being scattered to $k_{\|}=0$ and the other one on the upper branch. 


\section{Conclusion}

Angular resolved photoluminescence experiments allows getting a deeper understanding of the relaxation mechanism of microcavity polaritons under non-resonant excitation. We clearly evidence the collapse of a relaxation bottleneck resulting in a non-linear behavior of the emission close to $k_{\|}=0$. In a two-beam experiment, changes of the probe reflectivity and of the emission of large $k_{\|}$states evidence a stimulated scattering. The use of the two beams enables to locally create a large occupation factor while using a total carrier density far below the bleaching threshold.

We would like to acknowledge Bernard Sermage for very helpful discussions.

\section{References}

[1] C. Weisbuch, M. Nishioka, A. Ishikawa, Y. Arakawa, Phys. Rev. Lett. 69, 3314 (1992).

[2] For a review, see various early papers in: Confined Electrons and Photons, New Physics and Applications, Eds. E. Burstein, C. Weisbuch, NATO ASI Ser. B, Vol. 340, Plenum, New York 1995.

[3] A. Imamoglu, R.J. Ram, Phys. Lett. A 214, 193 (1996).

[4] F. Tassone, Y. Yamamoto, Phys. Rev. B 59, 10830 (1999).

[5] M. Kira, F. Jahnke, S.W. Koch, J.D. Berger, D.V. Wick, T.R. Nelson, G. Khitrova, H.M. Gibbs, Phys. Rev. Lett. 79, 5170 (1997).

[6] F. Jahnke, M. Kira, S.W. Koch, G. Khitrova, E.K. Lindmark, T.R. Nelson, D.V. Wick, J.D. Berger, O. Lyngnes, H.M. Gibbs, Phys. Rev. Lett. 77, 5257 (1996).

[7] R. Houdré, J.L. Gibernon, P. Pellandini, R.P. Stanley, U. Oesterle, C. Weisbuch, J. O'Gorman, B. Roycroft, M. Ilegems, Phys. Rev. B 52, 7810 (1995).

[8] L.S. Dang, D. Heger, R. Andre, F. Boeuf, R. Romestain, Phys. Rev. Lett. 81, 3920 (1998).

[9] J. Bleuse, F. Kany, A.P. de Boer, P.C.M. Christianen, R. Andre, H. Ulmer-Tuffigo, J. Cryst. Growth 184/185, 750 (1998).

[10] P. Senellart, J. Bloch, Phys. Rev. Lett. 82, 1233 (1999).

[11] A.I. Tartakovskii, V.D. Kulakovskii, D.N. Krizhanovskii, M.S. Skolnick, V.N. Astratov, A. Armitage, J.S. Roberts, Phys. Rev. B 60, R11293 (1999).

[12] P.G. Savvidis, J.J. Baumberg, R.M. Stevenson, M.S. Skolnick, D.M. Whittaker, J.S. Roberts, Phys. Rev. Lett. 84, 1547 (2000).

[13] R. Huang, F. Tassone, Y. Yamamoto, Phys. Rev. B 61, R7854 (2000).

[14] J. Erland, W. Langbein, J.R. Jensen, J.M. Hwam, to appear in Phys. Status Solidi.

[15] R. Houdré, C. Weisbuch, R.P. Stanley, U. Oesterle, M. Ilegems, private communication.

[16] C. Ciuti, P. Schwendimann, B. Deveaud, A. Quattropani, Phys. Rev B 62, R4825 (2000).

[17] R. Houdré, C. Weisbuch, R.P. Stanley, U. Oesterle, P. Pellandini, M. Ilegems, Phys. Rev. Lett. 73, 2043 (1994).

[18] P. Senellart, J. Bloch, B. Sermage, J.Y. Marzin, in preparation. 